\title{
ANXIETY AND DEPRESSION IN PATIENTS SUFFERING A MYOCARDIAL INFARCTION DURING THE COVID-19 PANDEMIC
}

\author{
Márcia Moura Schmidt ${ }^{1,2}$, Amanda Bittencourt Lopes da Silva ${ }^{1}$, \\ Samanta Fanfa Marques', \& Cynthia Seelig ${ }^{1}$ \\ IInstitute of Cardiology of Rio Grande do Sul (Brazil) \\ ${ }^{2}$ Graduate Program in Health Sciences: Cardiology (Brazil)
}

\begin{abstract}
Introduction: Acute myocardial infarction is an experience that may cause severe emotional impact to the individual who presented it. The anxiety and depression felt is caused not only by the infarction episode itself, but also by the current uncertain state of the world during and because of the COVID-19 pandemic, something which can heighten these negative feelings throughout the entire population, especially in those considered to be in high risk groups. Objective: The objective of the present study is to estimate the prevalence of anxiety and depression among patients infarcted during the current coronavirus pandemic in the state of Rio Grande do Sul. This study will compare clinical characteristics and risk factors between groups with and without anxiety and depression. Methods: Acute myocardial infarction patients who were submitted to percutaneous coronary intervention from February to June 2020 at a reference hospital in cardiology were considered eligible. The patients were interviewed via phone calls. The clinical characteristics and intra-hospital events were obtained from the hospital's REDCap Database. The level of anxiety and depression suffered was evaluated utilizing the HADS (Hospital Anxiety and Depression Scale). Patients considered as suffering from anxiety and depression were those who obtained scores for the possible and probable presence of those mood disorders. Patients were divided into groups with and without anxiety and depression. The categorical variables were expressed through frequency and percentage and analyzed by the Chi-square test and the continuous variables were described by average and standard deviation, and analyzed by Student's t-test. Results: A total of 55 patients were interviewed. The majority of these patients were male $(74,5 \%)$ and white $(80 \%)$. The average age of those participating in the study was $58 \pm 12$ years, and the female participants were older than the males. The average number of years of education was $8 \pm 4$ years. The prevalence of anxiety was $38,2 \%$ and of depression $30,9 \%$. Conclusions: The prevalence of anxiety and depression was higher than those described in the literature for this population, which supports the hypothesis that the pandemic may be aggravating the patient's emotional state. Another group will be interviewed for the control group.
\end{abstract}

Keywords: Anxiety, depression, myocardial infarction, COVID-19.

\section{Introduction}

Patients with heart disease generally experience negative psychological states (Mal, Awan, Ram, \& Shaukat, 2019). The acute myocardial infarction (MI), popularly known as heart attack, occurs due to thrombosis and sudden occlusion of a coronary artery due to atherosclerotic plaque rupture, fracture or erosion.

Despite the great medical advances in relation to the physical sequelae of post myocardial infarction (MI) patients, the sequelae psychological causes are still not fully recognized. MI causes a severe impact to the patient, significantly reducing the quality of life, increasing the subject's rehospitalization rate as well as their levels of stress and exhaustion (Kumar \& Nayak, 2017). Increasingly, attention is being paid to mood disorders in patients recovering from acute myocardial infarction, especially since depression was first reported to be associated with increased mortality in this group of patients.

Depression is three times more common in patients after a myocardial infarction than those who have never suffered one (Thombs, Bass, Ford, Stewart, Tsilidis, Patel,Fauerbach, Bush \& Ziegelstein , 2006). A studies reported that about $31 \%$ of patients in post-MI experience anxiety, with women presenting a higher incidence (Kumar \& Nayak, 2017). 
Generally, in the event of a pandemic, people's physical health and the fight against the pathogenic agent are the primary focus of attention for managers and health professionals (Ferguson, Laydon, Nedjati Gilani, Imai, Ainslie, Baguelin \& Ghani, 2020). However, measures that may reduce the psychological impacts of the pandemic cannot be overlooked at this time (Wang, Pan, Wan, Tan, Xu, Ho \& Ho, 2020).

\section{Objective}

The present study aims to estimate the prevalence of anxiety and depression on patients infarcted during the current COVID-19 pandemic at Institute of Cardiology of Rio Grande do Sul, by comparing clinical characteristics and risk factors between groups with and without anxiety and depression.

\section{Methods}

\subsection{Inclusion and exclusion criteria}

Cross-sectional study with post-myocardial infarction patients, according to the V Guideline of the Brazilian Society of Cardiology on the treatment of acute myocardial infarction with ST segment elevation (2015). Exclusion Criteria: Previous documented history of dementia, cognitive difficulties, or psychiatric diagnosis.

\subsection{Logistics}

Infarcted patients were contacted between February and June 2020, via telephone calls, in order to explain the purpose of the present study. The interviews were carried out after the consent form was read to the patient and the patient consented to participate. The patient sent an SMS message with the word "yes" to the researchers' phones, expressing their agreement to participate in the study.

\subsection{Assessment}

The instrument used was the HADS (Hospital Anxiety and depression Scale), a scale translated and validated by Botega, Bio, Zomignani, Garcia Jr. and Pereira (1995). The goal is to screen mild degrees of affective disorders in non-psychiatric environments and that is why it has been used in patients with organic illness. It consists of 14 multiple choice items, seven of which are aimed at assessing the anxiety (HADS-A) and seven for assessing depression (HADS-D). Each item can be scored from 0 to 3, reaching a maximum of 21 points in each subscale. Scores of 8 to 11 points denote possible and from 12 to 21 denote probable cases of anxiety or depression. In this study, the cut-off points assumed was a score equal to or greater than 8 .

\subsection{Statistical analysis}

The sample calculation was performed in the WINPEPI program, version 11.65 , considering a power of $80 \%$ and a significance level of $5 \%$ and a proportion of 0.6 between the groups with and without depression. The data were based on the article by Tombs (2006) that showed several prevalence's of depression in studies with the HADS scale. There is a variation of $11 \%$ to $17 \%$ in one study and, in another, $15.5 \%$ (CI-13 to $18 \%$ ). So, considering a 0.6 ratio between depressed and non-depressed, 49 participants were required. The data were analyzed using SPSS software, version 26.0. Continuous variables were presented as mean and standard deviation and categorical variables using frequency. They were compared using the Student's t-test or Chi-square.

\subsection{Ethical and legal considerations}

This protocol is in accordance with the Helsinki declaration and was approved by the local ethics committee.

\section{Results}

Fifty-five patients who responded to the survey were analyzed. Of these patients, the majority were male $(74.5 \%)$ and white $(80 \%)$. The mean age of the sample was $58.65 \pm 12.24$ years, with females being older than males. The average number of years of education of the participants is $7.83 \pm 3.63$ years and the average of ejection fraction was $49.92 \% \pm 15.32 \%$.

In this sample group, $38.2 \%$ of the infarcted patients presented anxiety, and $30.9 \%$ presented depression during the current COVID-19 pandemic. 
Table 1 shows the presence of anxiety and depression according to risk factors. Patients with chronic renal failure, trivascular coronary lesions and those who were being treated with acetylsalicylic acid displayed a greater tendency to anxiety. With respect to depression, the levels of depression were higher in those who use acetylsalicylic acid. We observed a higher percentage of females in the anxious and depressed group than males, but the difference between the two was not statistically significant.

Table 1. Anxiety and depression rates according to previous history.

\begin{tabular}{lcccccc} 
& Anxiety & No Anxiety & $\boldsymbol{p}$ & Depression & $\begin{array}{c}\text { No } \\
\text { Depression }\end{array}$ & $\boldsymbol{p}$ \\
\hline $\begin{array}{l}\text { Sexo } \\
\text { Male }\end{array}$ & & & & & & \\
Female & $36.60 \%$ & $63.40 \%$ & 0.677 & $29.30 \%$ & $70.70 \%$ & 0.652 \\
Risk Factors & $42.90 \%$ & $57.10 \%$ & 0.677 & $35.70 \%$ & $64.30 \%$ & 0.652 \\
Smoking & & & & & \\
Hypertension & $42.90 \%$ & $41.20 \%$ & 0.948 & $41.20 \%$ & $42.10 \%$ & 0.304 \\
Diabetes & $47.10 \%$ & $50.00 \%$ & 0.389 & $47.10 \%$ & $57.90 \%$ & 0.456 \\
Dyslipidemia & $33.30 \%$ & $44.10 \%$ & 0.428 & $41.20 \%$ & $39.50 \%$ & 0.905 \\
Premature Family History for & $9.50 \%$ & $29.40 \%$ & 0.083 & $11.80 \%$ & $26.30 \%$ & 0.227 \\
$\quad$ CAD & $19.00 \%$ & $21.00 \%$ & 0.890 & $18.00 \%$ & $21.00 \%$ & 0.770 \\
Cronic Kidney Falirure & $9.50 \%$ & $0.00 \%$ & $\mathbf{0 . 0 6 7}$ & $5.90 \%$ & $2.60 \%$ & 0.552 \\
Acetylsalicylic treatment & $28.60 \%$ & $9.10 \%$ & $\mathbf{0 . 0 6 1}$ & $28.60 \%$ & $16.20 \%$ & $\mathbf{0 . 0 6 1}$ \\
$\quad$ Three vessel disease & $23.80 \%$ & $15.60 \%$ & $\mathbf{0 . 0 8 8}$ & $23.50 \%$ & $16.70 \%$ & 0.134 \\
\hline
\end{tabular}

Regarding the HADS scale, it was observed that that the patients suffering from anxiety felt tense and wound up for a significant period of time compared with those not suffering from anxiety (42.9\% vs $8.8 \%)$. Among those suffering from depression, the majority $(58.9 \%$ vs $5.3 \%)$ felt pleasure occasionally while watching or listening to a TV or radio program or while reading something. All questions were statistically significant among participants with and without anxiety and depression, with the exception of one item on the depression scale which affirmed: "I feel as if I am slowed down" (Figure 1).

Figure 1. HADS Scale response example.

I get a sort of frightened feeling like 'butterflies' in the stomach

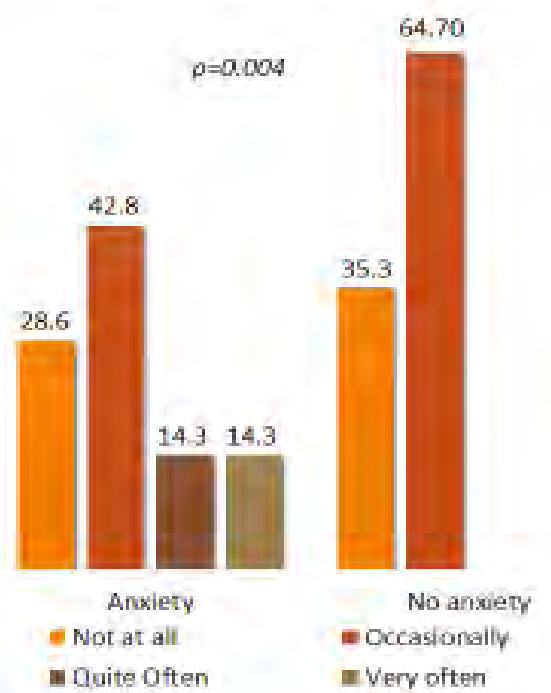

I feel as if I am slowed down

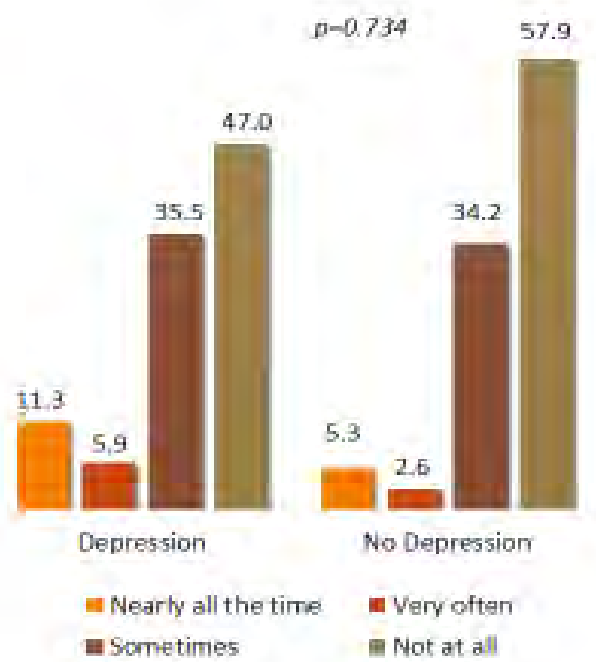




\section{Discussion}

In this study we found $38.2 \%$ of the infarcted patients displayed anxiety, and $30.9 \%$ displayed depression during the current COVID-19 pandemic. The study conducted by Kala in which he evaluated patients after the infarction episode, found three months after AMI, 10.4\% had levels of depression and $4.5 \%$ of anxiety (Kala, Hudakova, Jurajda, Kasparek, Ustohal, Parenica \& Kanovsky, 2016). In another study that measured the presence of anxiety and depression in infarcted patients using HADS, it was found that 23.3\% of patients had anxiety and 18.6\% depression (Larsen, Christensen, Nielsen \& Vestergaard, 2014). Both studies displayed a lower prevalence of anxiety and depression than that was found in this study.

It was also possible to perceive a greater tendency towards anxiety in patients with chronic renal failure, three-vessel disease and those in continuous treatment with acetylsalicylic. The emotional state of patients with chronic diseases has been extensively studied (Lebel, Mutsaers, Tomei, Leclair, Jones, Petricone-Westwood, Rutkowski, Ta, Trudel, Laflamme, Lavigne \& Dinkel, 2020) Since they have to deal with the persistence and unpredictability of their illness, they often report feeling anxious and worried. With respect to depression, the levels of depression were higher in those who use acetylsalicylic acid, which may indicate chronic heart disease, as shown in the literature the association between depression and comorbidities (Carvalho, Bertolli, Paiva, Rossi, Dantas, \& Pompeo, 2016). It was observed that women tend to suffer more from anxiety and depression when compared to men, which is in accordance with the literature (Tombs et al., 2006). In this study, statistical significance was not reached but this may be due to the sample size and the number of women in the study, which is always fewer than that of men in terms of heart disease.

As we found all the statistically significant issues among the participants with and without anxiety and depression, we can assume that the test is valid to dignify them from the cut-off point of 8 , which proposes to classify the subjects with possible anxiety or depression. The screening process was adequate and provided sufficient suitable candidates. With reference to "I feel as if I am slowed down", this was not significant as it may be a common symptom in post-infarction patients, due to the fear of overexertion and suffering another infarction or some other complication.

The experience of acute myocardial infarction is often traumatic for the subject and may have sequelae in addition to the physical. The COVID-19 pandemic appears as yet another risk factor for mood disorders, as the population has seen their lives change completely from one day to the next: physical and social isolation, unemployment, constant fear of an unknown and potentially fatal virus. We found prevalences of anxiety and depression higher than those described in the literature for this population, which corroborates the hypothesis that the pandemic may be aggravating the emotional state of patients after a heart attack.

This study has some limitations, such as: the small number of participants and the absence of a control group. What is presented here are partial results only however these data made it possible to establish psychological post infarction care routines, reducing the risks of psychological factors. As for future perspectives, for control purposes, we intend to interview a group between 55 and 75 years old, none of whom suffer from cardiovascular diseases.

\section{Conclusion}

Post-infarction patients often suffer from anxiety and depression. During the pandemic, we found a tendency among those with chronic kidney disease, trivascular coronary injury and previous treatment with acetylsalicylic acid to be more anxious. Also those who were already using this medication were more prone to depression. The prevalence of anxiety and depression found in this sample of post-infarction patients is higher than that described in the literature. A control group will be evaluated.

\section{References}

Botega, N. J., Bio, M. R., Zomignani, M. A., Garcia Jr, C., \& Pereira, W. A. (1995). Mood Disorders Among Inpatients in Ambulatory and Validation of the Anxiety and Depression Scale Had [transtornos Do Humor Em Enfermaria De Clínica Médica E Validação De Escala De Medida (HAD) De Ansiedade E Depressão.]. Public Health Magazine, 29, 359-363.

Carvalho, I. G., Bertolli, E. D. S., Paiva, L., Rossi, L. A., Dantas, R. A. S., \& Pompeo, D. A. (2016). Anxiety, depression, resilience and self-esteem in individuals with cardiovascular diseases. Latin American Journal of Nursing, v.24. 
Ferguson, N., Laydon, D., Nedjati Gilani, G., Imai, N., Ainslie, K., Baguelin, M., ... \& Ghani, A. (2020). Report 9: Impact of non-pharmaceutical interventions (NPIs) to reduce COVID19 mortality and healthcare demand. Imperial College London. DOI: https://doi.org/10.25561/77482.

Kala, P., Hudakova, N., Jurajda, M., Kasparek, T., Ustohal, L., Parenica, J., ... \& Kanovsky, J. (2016). Depression and anxiety after acute myocardial infarction treated by primary PCI. PLoS One, 11(4): 1-9. DOI:10.1371/journal.pone.0152367

Kumar, M., \& Nayak, P. K. (2017). Psychological sequelae of myocardial infarction. Biomedicine \& Pharmacotherapy, 95, 487-496.

Larsen, K. K., Christensen, B., Nielsen, T. J., \& Vestergaard, M. (2014). Post-Myocardial Infarction Anxiety or Depressive Symptoms and Risk of New Cardiovascular Events or Death: A Population-Based Longitudinal Study. PSYCHOSOMATIC MEDICINE, 76(9): 739-746.

Lebel, S., Mutsaers, B., Tomei, C., Leclair, C.S., Jones, G., Petricone-Westwood, D., Rutkowski, N., Ta, V., Trudel, G., Laflamme, S.Z., Lavigne, A.A., Dinkel, A. (2020). Health anxiety and illness-related fears across diverse chronic illnesses: A systematic review on conceptualization, measurement, prevalence, course, and correlates. PLOS ONE, 15(7):1-48.

Mal, K., Awan, I. D., Ram, J., \& Shaukat, F. (2019). Depression and Anxiety as a Risk Factor for Myocardial Infarction. CUREUS, 11(11): 1-5. DOI: 10.7759 / cureus.6064

Sociedade Brasileira de Cardiologia. V Diretriz deTratamento do Infarto Agudo do Miocárdio com Supradesnível do Segmento ST. Arquivos Brasileiros de Cardiologia, 2015; 105(2), 1-121.

Thombs, B. D., Bass, E. B., Ford, D. E., Stewart, K. J., Tsilidis, K. K., Patel, U., ... \& Ziegelstein, R. C. (2006). Prevalence of depression in survivors of acute myocardial infarction. Journal of General Internal Medicine, 21(1), 30-38.

Wang, C., Pan, R., Wan, X., Tan, Y., Xu, L., Ho, C. S., \& Ho, R. C. (2020). Immediate psychological responses and associated factors during the initial stage of the 2019 coronavirus disease (COVID-19) epidemic among the general population in China. International Journal of Environmental Research and Public Health, 17(5), 1729: 1-25. 